\title{
Nanotechnology: Emerging Developments and Early Detection of Cancer A Two-Day Workshop sponsored by the National Cancer Institute and the National Institute of Standards and Technology, August 30-31 2001, on the National Institute of Standards and Technology Campus, Gaithersburg, MD, USA
}

\author{
Steven J. Zullo ${ }^{\mathrm{a}}$, Sudhir Srivastava ${ }^{\mathrm{b}}$, J. Patrick Looney ${ }^{\mathrm{c}}$ and Peter E. Barker ${ }^{\mathrm{d}}$ \\ ${ }^{a}$ Chemistry and Life Sciences Division, Advanced Technology Program, National Institute of Standards and \\ Technology, Gaithersburg, MD 20899-4730, USA \\ ${ }^{\mathrm{b}}$ Cancer Biomarkers Research Group, Division of Cancer Prevention, National Cancer Institute, 6130 Executive \\ Plaza North, Suite 3142, Bethesda, MD 20892, USA \\ ${ }^{\mathrm{c}}$ Program Office, National Institute of Standards and Technology, Gaithersburg, Md 20899-8311, USA \\ ${ }^{\mathrm{d}}$ NIST-NCI Biomarkers Validation Laboratory, DNA Technology Group/Biotechnology Division, National Institute \\ of Standards and Technology, 100 Bureau Drive, Gaithersburg, MD 20899-8311, USA
}

\begin{abstract}
A recent meeting jointly sponsored by the National Cancer Institute (NCI) and National Institute of Standards and Technology (NIST) brought together researchers active in nanotechnology and cancer molecular biology to discuss and evaluate the interface between disciplines. Emerging areas where nanotechnologies may impact cancer prevention and early cancer detection were elaborated by key researchers who catalyzed interdisciplinary dialogue aimed at fostering cross-discipline communications and future collaboration.
\end{abstract}

Keywords: Biomarker, cancer detection, nanotechnology, high throughput, microarray, mitochondria

\section{Meeting Report}

At the workshop (August 30-31, 2001) organized by the National Cancer Institute (NCI) and the Na- tional Institute of Standards and Technology (NIST) on "Nanotechnology in Early Detection of Cancer", nanotechnology experts from academia, industry and government institutions discussed state of the art tech- 
nologies and evaluated current and prospective applications of nanotechnology for early cancer detection. Experts from both cancer research and nanotechnology presented examples from their own research and commented on potential impact of novel nano-scale tools as applied to cancer research.

The workshop of cancer researchers, molecular biologists, chemists, physicists, nanotechnologists, and other scientists, convened at the NIST campus, was jointly sponsored by the Cancer Biomarkers Research Group of the Division of Cancer Prevention (DCP) of the NCI, the DNA Technologies Group/ Chemical Sciences and Technology Laboratory (CSTL) of NIST and the NIST Advanced Technology program (ATP Program). Dr. Lee Hood, President and Director, Institute for Systems Biology, chaired the session Nanotechnology Devices for Early Detection of Cancer, and Dr. George Whitesides, Professor, Department of Chemistry and Chemical Biology, Harvard University, chaired Nanotechnology Platforms for High Throughput Identification of Biomarkers.

Nanotechnology is defined as the creation of functional materials, devices and systems through control of matter at the scale of 1 to 100 nanometers, and exploitation of novel properties and phenomena at the same scale. Advances in nanotechnology are the impetus for the "Next Industrial Revolution" by the National Nanotechnology Initiative (NNI). One potential application highlighted in the NNI is the detection of diseases. This might obviate patient care with a shift towards early detection and prevention.

The purpose of the workshop was to determine how and when advances in nanotechnology could be applied to the early detection of cancer. Topics included lasers to measure optical deformability in cancer cells, detection, sensing and therapeutics through sensors based on nanopores and nanomaterials, molecular combing to detect genomic instability, molecular nanomechanics for detection of biomolecular interactions, dendrimers, nanodevices and nanotechnology platforms for sensing, delivery and therapeutic applications. The Workshop highlighted the potential nanotechnology has to make significant contributions to cancer prevention and detection in addition to diagnosis and treatment. The consensus was that, nanotechnology offers important new tools for detection at a time when existing more conventional technologies might be approaching their limits. Nanotechnology was identified as a possible means to provide direct readout of genomic and proteomic information both at the single-cell and singlemolecule level. Its utility in analyzing and characteriz- ing extremely limiting amounts of biological material was also explored. This commentary summarizes the highlights of the workshop and the recommendations made by the leading proponents of nanotechnology.

Dr. Peter Greenwald (Director, Division of Cancer Prevention, National Cancer Institute) described DCP goals that could be reached with the help of advances in nanotechnology: (1) research on biomarkers, and (2) clinical trials in early detection and prevention of cancer, with the aims of reducing the incidence and mortality rates of cancer. Nanotechnology offers to help refine early detection and diagnostics to provide endpoints for cancer prevention and help drive improvements in cancer therapy.

Dr. Hratch Semerjian (Director, CSTL) described biotechnology-related research conducted in NIST laboratories, particularly in the NIST Biotechnology Division, including the Center for Advanced Research in Biotechnology (CARB), a joint University of Maryland-NIST endeavor. Dr. Semerjian described the long history of collaboration between NIST and the $\mathrm{NCI}$, especially in the area of clinical laboratory quality assurance programs. He noted that this meeting was being held on the first anniversary of the interagency agreement on biomarker validation that involves NIST and the NCI.

\section{NCI Vision of Nanotechnology}

Cancer is a series of diseases rather than one single disease. All of these diseases involve changes in the genetic code of cells, resulting in altered expression of gene products, RNA, and protein. Protein interactions are critical to the genesis of the cancerous cells and to the response to therapy and interventions. Understanding these critical interactions enables researchers to develop the technologies needed to take the next steps in the discovery process.

Cancer currently is being missed at its earliest stages, in part because detection methods are not directed at cellular changes of carcinogenesis. The NCI is encouraging discovery and elaboration of those early cellular changes. The NCI will further derive information on biomarkers as well as define significant opportunities to define diagnostically useful signatures for determining the next step of therapy, treatment, or prevention. NCI is also supporting identification of new biological targets for treatment and prevention at the molecular level. To shift from therapeutic to a preventive mode, a new nano-scale tool kit is needed to: (1) detect biomark- 
ers, signatures, and targets; (2) determine their best uses; (3) apply them in the early stages of cancer development; and (4) measure, analyze, and manipulate molecular processes at scale and in context.

Nanotechnology will help define cancers by molecular signatures denoting processes that reflect fundamental changes in cells and tissues that lead to cancer. To support the measurement, analysis, and manipulation of molecular processes at scale and in context, new technologies will be needed. These ultimately will become the first wave of clinical tools to examine tissues and samples, pushing towards an entirely new method of addressing cancer that integrates detection, diagnosis, and intervention on a common technology platform. A series of questions arose: (1) Can nanotechnology enable the cancer research community to meet the challenges associated with early detection, (2) What are the promising novel technologies, (3) How can the integration of nanotechnology development in cancer research be encouraged and fostered, and (4) How can the cancer and nanotechnology research communities be brought together?

There are a number of NIH-sponsored programs that support development of new technologies for this tool kit, including the Innovative Molecular Analysis Technologies Program (IMAT) (URL: http://otir.nci.nih. gov/tech/imat.html). Advancing the technology of early cancer detection to enable new applications and allow for higher throughput at reduced cost requires increased automation, increased parallel processing, and miniaturization with reductions in scale. In developing molecular analysis technologies, the push to reduce scale has proceeded through the range of microdynamics and microfluidics into the realm of nanotechnology. It is hoped that this push will extend into detection, giving clinicians and researchers the opportunity to detect cancerous processes inside the body at an early stage in a noninvasive or minimally invasive manner. Advances in nanotechnology hopefully will allow researchers to develop biomolecular sensors that will enable informative feedback loops to: (1) find early signatures of disease; (2) diagnose what intervention is needed; and (3) determine the effectiveness of treatments.

The trend toward technology reductions of size approaching the nano-scale is driven by the advantage of working in a size context compatible with use in the human body. Nano-technological advances have expanded understanding of the structure, function, and relationships of biological macromolecules as well as the creation of new devices that have the capabilities for producing functional ma- terials at scale, although their role is still emerging. Three NCI-sponsored programs that are utilizing nanotechnological advances in biomarker experiments include the Unconventional Innovations Program (URL: http://otir.nci.nih.gov/tech/uip.html ); the NCI/National Aeronautics and Space Administration Collaboration on Biomolecular Sensors (URL: http://nasa-nci.arc.nasa.gov/), and the NCI Early Detection Research Network(EDRN)(URL: http://www3. cancer.gov/prevention/cbrg/edrn/).

\section{NIST Activities in Nanotechnology}

NIST laboratories are organized primarily by discipline, although many NIST programs are interdisciplinary and span laboratories. The primary role of NIST is in measurement science and measurements of fundamental physical phenomena. Thus nanotechnology presents tremendous challenges to ongoing research and applications at NIST. The NIST role in nanotechnology is to develop measurements and standards that will guide and expedite the development of new nanotechnologies. As manufacturing nano-devices develops, NIST will help develop benchmark measurements that will be critical for understanding the performance of nano-devices and nanoscale phenomena.

Current NIST nano-scale activities include work in photolithography, molecular electronics, materials characterization, chemical imaging, and biological measurements. For example, NIST recently developed a nano-scale physics facility, at which researchers fabricate structures on surfaces under strictly controlled conditions and ask fundamental questions about what happens when one or more atoms are put together. This work ranges from quantum mechanical phenomena to macroscopic phenomena, and holds the potential for new science to emerge as novel capabilities are developed in response to newly established benchmarks. Private sector firms, often teamed with university collaborators, are funded by the Advanced Technology Program (ATP) to conduct much of the extramural NIST nanotechnology research. ATP supports a wide range of innovative, advanced technologies via competitive peer-reviewed government-industry costshare awards. These include nano-structured materials, nano-diagnostics, and nano-fabrication. In the most recently completed competition (Fiscal Year 2001), awards were made for nano-scale technologies (http://www.atp.nist.gov/awards/2001list.htm). 


\section{Nanotechnology Devices for Early Detection of Cancer}

Dr. Hood commented that cancer diagnosis, cancer therapy, and cancer prevention must be closely integrated, as all deal with similar sets of biological information. This will lead to individualized medicine in cancer diagnosis and treatment. Thus, there are different dimensions of stratification and progression that researchers must address. Prostate cancer, for example, may have three or four biologically different stages, each with the different ability to progress. Multiparameter analyses can be used to distinguish these two processes from each other. The ability to comprehensively profile proteins in blood would be a tremendous advance. However, a significant challenge to highprecision multi-parameter analyses in blood is dealing with large amounts of serum albumin. DNA arrays combined with nano-devices can identify molecules that are tissue-specific, which would allow for the identification of tissue-specific markers for diagnostic purposes and ultimately, create an antibody molecule that activates the innate immune system. Nanotechnology offers the ability to interrogate cells, in blood, and in biopsies in the future. The nanotechnology approach to diagnostics and therapeutics combines genomics, proteomics, protein engineering, and cellular manipulation. Nanotechnology opportunities emerging for cancer diagnostics, therapeutics, and prevention will involve single-molecule analysis, single-cell analysis, small cell populations, and multi-parameter analysis. Microfluidics and microelectronics will provide key and striking opportunities, and these technologies will move down in scale to the nano-realm. With the PSA test (prostate specific antigen, or kallikrein 3), cancer can be identified relatively early. With nanotechnology multiple biopsies may become possible, and with this the ability to analyze specific and multiple analytes in vivo.

Nanotechnology provides new ways to decipher biological information. One possibility is application of nanopores to sequence DNA and characterize proteins and other molecules. The ability to genotype at very high throughput will be exceptionally important. Meeting participants noted that it might be necessary to examine 100,000-500,000 genetic markers in an individual to carry out a comprehensive sophisticated detailed genetic analysis. Current conventional techniques are expensive, costing up to $\$ 1.50$ per marker. Dr. Hood noted that nanotechnology offers possibilities for detection of RNA splicing, examining proteins, and as- saying cellular behaviors at the level of single cells. He closed his remarks by stating that members of the breakout group felt strongly that the progression of micro-fluidics and micro-electronics into nanotechnology is a powerful and exciting development. This development has potential to transform the areas of cancer diagnostics, therapeutics, and prevention.

To be useful, technology must move towards increased miniaturization, offer parallel analyses and integration in an automated system. Particular opportunities will offer a view of single molecules, single cells, and in small samples. Multiplex analyses will be important; and all the advantages of micro-fluidics, microstructure, and nanotechnology will be needed. For example, in prostate cancer, there can be two different types of prostate cancer cells. In one, the localized cancer cell is virtually identical to a luminal cell, while in metastatic cancer, the cell is virtually identical by phenotype to normal stem cells. There are different cell surface markers on these two cell types. It is possible to identify a whole series of cell surface markers

Researchers, when developing technology at the nanometer scale, should bear in mind dynamic range, techniques for RNA expression and RNA splicing. Particularly in cancer, detection methods capitalize on amplification technologies, while proteomics initiatives are disadvantaged by the lack of appropriate technology for protein amplification, although phage display technologies do offer potential in that the detection molecule can be amplified. In this and other approaches, sensitivity of detection is preeminent.

Thus, nanotechnology may enable detection of single cell phenotype. Indeed, analysis, diagnosis, treatment, and ultimately prevention are all interrelated. The current approach begins with microarrays to interrogate multiple tissues to determine specific genetically determined characteristics, or cellular phenotypes. The power of proteomics is then brought to bear on the system under examination, for example, by finding a tissue specific cell surface molecule against which to raise an antibody to detect the errant cell. The ultimate goal then of the genomics, proteomics, protein engineering and cellular manipulation, is to activate the innate immune system, utilizing the molecular specificity and engineering of the living organism in a coherent integrated systems approach to diagnosis and treatment of cancer. 


\section{Nanotechnology Platforms for High Throughput Identification of Biomarkers}

Pursuing advances in nanotechnology is worthwhile, as the research science holds potential for significant technical contributions to the prevention, detection, diagnosis, and treatment of cancer. Dr. Whitesides noted that these are areas in which tools are desperately needed, and mechanisms to support the development of tools should be improved, particularly on the part of the NIH. The support roles of the NIST intramural laboratories, and the extramural ATP, also address and promote this expressed need for nanotechnology tool development.

Nanotechnology holds promise for providing new tools for understanding the cell, the differences between normal and abnormal cells, and the mechanisms of communication between them. This information is fundamental to design of cancer detection and prevention strategies. Nanotechnology may also provide nondestructive "windows" into cells, with the ability to manufacture particles or probes that are small enough to be inserted into cells and monitor in real time without damaging the cell. Nanotechnology offers the chance to explore the mechanical properties of the cell, including energy metabolism, morphology, cytoskeletal development, internal fluid flows, transport of components, and signaling pathways. In diagnosis and treatment, single cell analysis, characterization of tumor margins, isolation of rare cells from blood, and new tools for imaging may be possible with advances in nanotechnology. Dr. Whitesides noted that it would be difficult to determine whom to screen with this new technology, if it develops to an appropriate level.

One of the major challenges to developing this potential synergy is finding ways to bring the physics and engineering communities together with biologists and clinicians in a more productive social milieu. There is a fundamental distinction between the physical sciences, which tend to be mathematical and quantitative, and the biological sciences, which tend to be non-quantitative. Institutional processes that bring all of these types of scientists together are needed, but there is no clear consensus on how to achieve this. Systems that require these disciplines and individuals to work together collectively may be required. Dr. Whitesides added that education is key. These groups will have to learn new vocabularies of other scientific fields and understand their strengths and weaknesses. Workshops, exchanges of students and more senior researchers, and better access to published literature may help address the cul- tural divide between biology and engineering. Also, there is need of more effective information dissemination explaining the specialized NIH programs available to researchers, especially those in engineering and physics. There is a general perception among the science and engineering communities that the hypothesisdriven NIH is not receptive to the needs of technology developers. This perception needs to change on both sides. Steps must be taken to ensure that this occurs, so that the NIH can support new technologies earlier in their development.

Dr. Whitesides commented that one technology that has made the difference in the approach to cancer, indeed to biology, has been the technology of arrays, a technology not initially supported by NIH. The NIST ATP funded a number of companies that were early major players in developing the microarray technology.

\section{Discussion of Meeting Results}

Dr. Hood elaborated six paradigm shifts in biology that have resulted from the human genome project, and how these changes may affect the development of nanotechnology.

1. Discovery science. The challenge "discovery science" raises is how discovery can and should be integrated with hypothesis-driven research. This will be a challenging mandate for academic institutions if they are to take advantage of the advances resulting from the human genome project, and potentially, from nanotechnology.

2. Biology is, at its core, information science. There are two basic kinds of information in the genome: information contained in the genes, and information in the regulatory networks that specify how the genes operate. One of the key and primary intellectual challenges of the 21 st century will be solving the question of regulatory networks. This understanding is central to evolution, development, and complicated physiologic mechanisms. Biological information is hierarchically organized. To fully understand living systems, the capability to integrate the different types of hierarchical information is crucial.

3. High throughput biology. From the first capillary sequencer in 1986 to the DNA sequencing instruments of today, there has been a 2,000-fold increase in throughput, an increase in the quality of the sequence data, and a decrease in the cost 
per base pair. Those changes have all come as a result of incremental improvements in chemistry, engineering, and software. The next generation of instruments likely will be microfluidics-based.

4. Accrual to biology of the tools for mathematics and statistics. Biology is unique in that it is the only science whose core information is digital in nature. In more than 4 billion years of evolution, organisms have evolved to encode digital information in an incredibly complex, efficient and hierarchical manner. Unraveling this will have interesting implications.

5. Model organisms have been developed. These are critical in deciphering biological complexity and common, evolutionarily conserved biological themes. They do so via: (1) a genetics parts list, and (2) the ability to limit the parts list and examine the regulatory networks while starting to decipher the logic of life for a given organism. Comparative genomics will be a very powerful tool and an incredibly important one, because evolution is not well understood. Evolutionary mechanisms, once unraveled, provide the rationale for biological design.

6. The genome has provided access to human variability. The genome has allowed access to polymorphisms, the variations that distinguish individuals from one another, a very small fraction of which predispose to diseases such as cancer. The science now is in a reactive medicine phase, but it is moving towards a predictive phase. For some diseases and disorders, researchers can begin to develop detailed probabilistic histories, and hopefully, generate preventive therapies.

Each of these paradigm shifts is facilitated by a systems biology approach. The concept behind systems biology is that biological systems can be interrogated not one gene or one protein at a time, but from the context of how all genes or all proteins behave. According to Dr. Hood, the field of biology appears to be going through a series of paradigm changes that are fundamentally different from previous changes to the field. Biomedical science needs to change and co-evolve as well. Three suggestions were made: (1) change the education of students and physicians; (2) emphasize tool development, because shifting from predictive to preventive medicine will require a vast array of new global technologies; and (3) marry the bioengineering, computer science, engineering, and physical science communities, with biology, so those cross-disciplinary scientists can gain a deep understanding of what the problems are and focus on them. Also, it was suggested that the scientists should be allowed to take larger steps and gamble on developing technologies.

A predominant view of the workshop is that the fundamental progress of the NIH depends on the development of tools so that researchers can explore hypotheses that previously were intractable before nanotechnology. It was mentioned that the NIH should be reaching out to researchers to a greater degree. Participants discussed the role of the ATP and the interplay between private industry, academia, and the government in terms of matching funds and intellectual property in bringing the day and a half activity to a close.

\section{Acknowledgements}

The authors wish to thank the ATP and the DNA Technologies Group at NIST, and the Division of Cancer Prevention at NCI for supporting this meeting.

\section{About the Authors}

Steven J. Zullo is Project Officer with the Chemistry and Life Sciences Division of the NIST Advanced Technology Program. Sudhir Srivastava is the Chief, Cancer Biomarkers Group, Division of Cancer Prevention, National Cancer Institute. Dr. Srivastava directs the DCP program Early Detection Cancer Network (EDRN). Patrick Looney is a Physicist with the Program Office, Office of the Director at NIST; Peter Barker is Biologist and Project Leader at the DNA Technologies Group, Biotechnology Division of CSTL at NIST. 


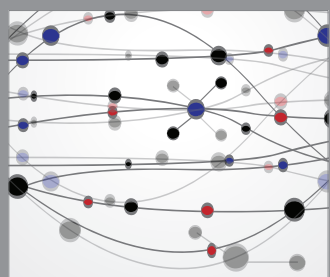

The Scientific World Journal
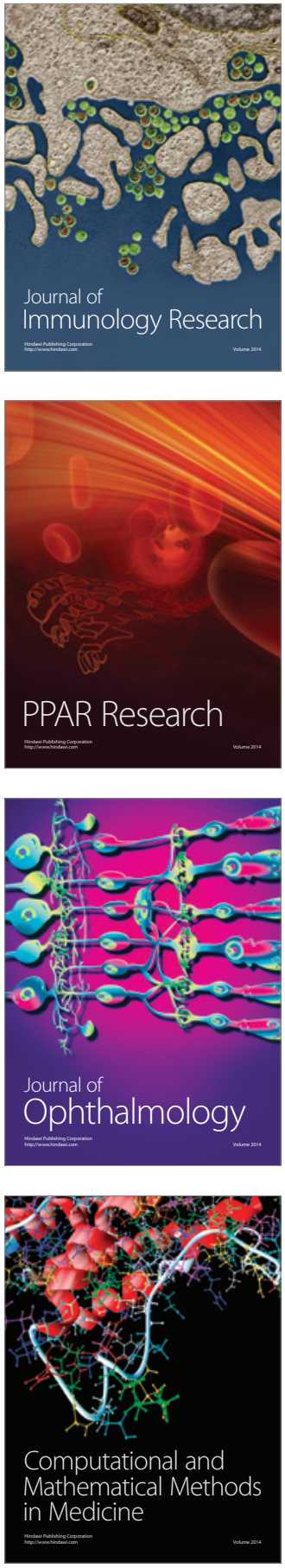

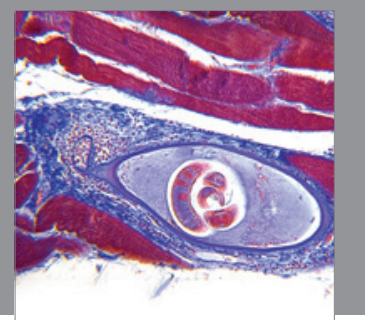

Gastroenterology

Research and Practice
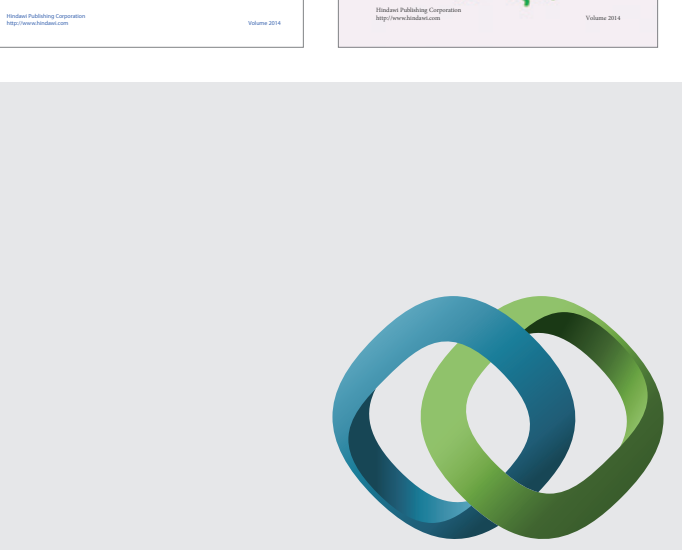

\section{Hindawi}

Submit your manuscripts at

http://www.hindawi.com
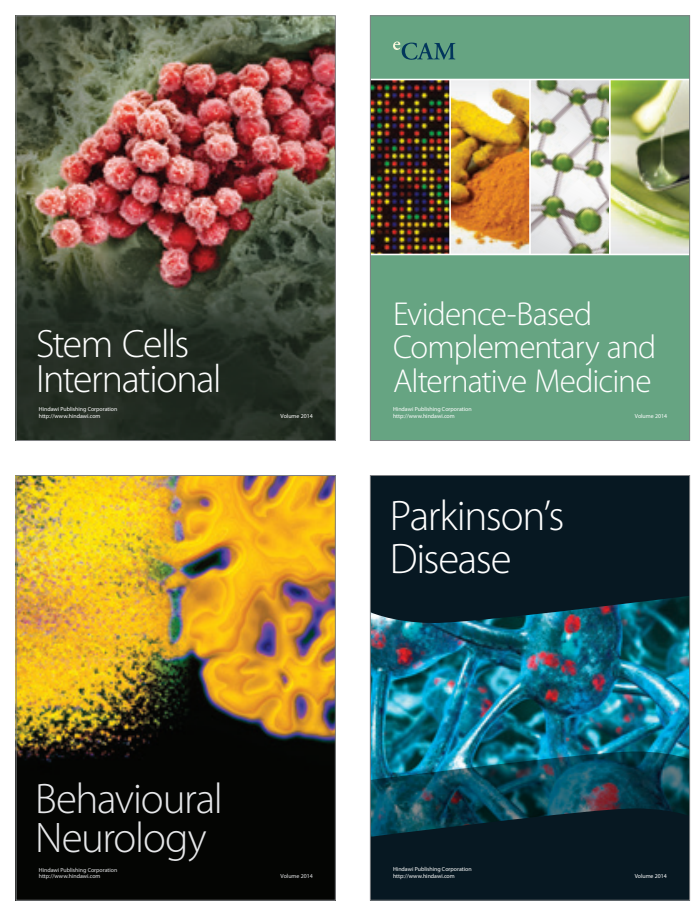

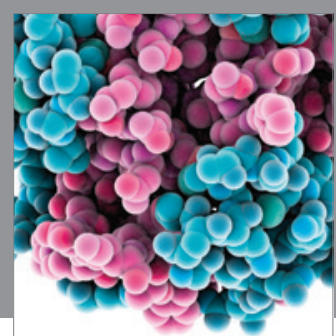

Journal of
Diabetes Research

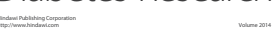

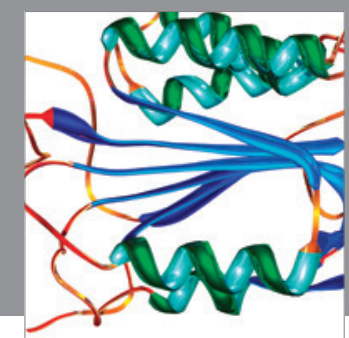

Disease Markers
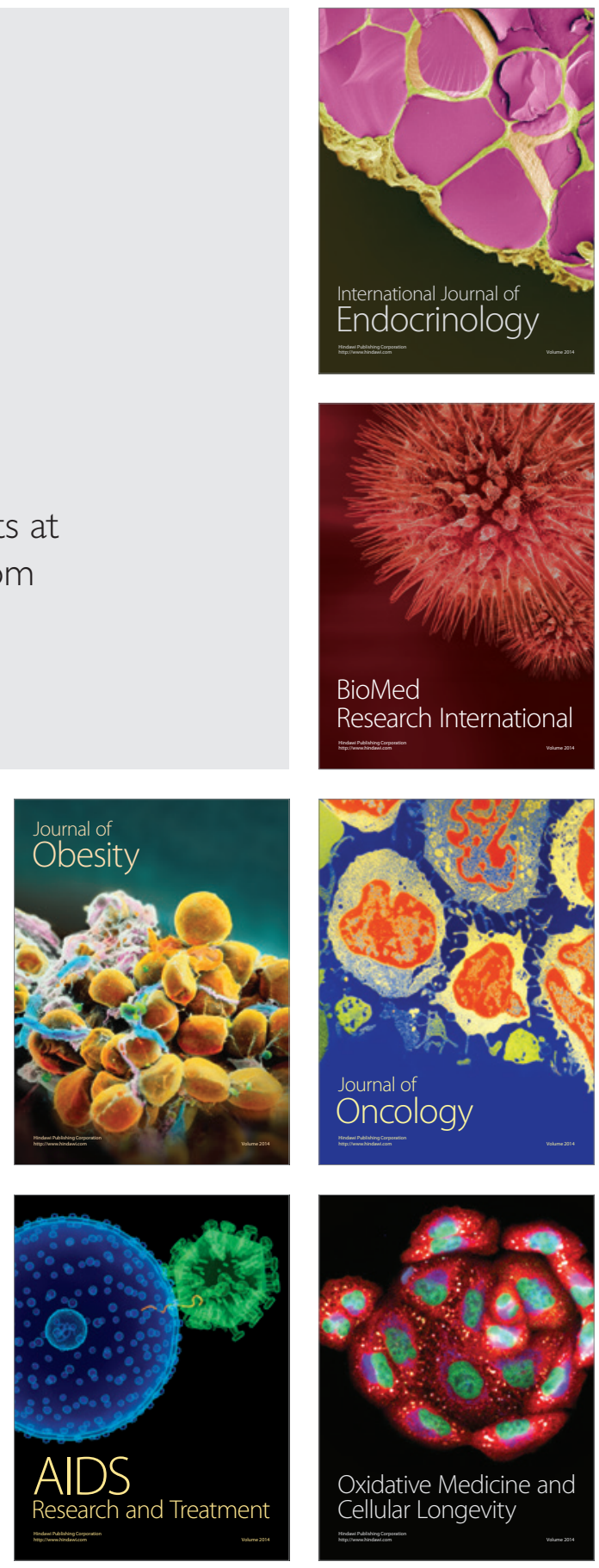